\title{
Gross anatomical characterization of the psoas major muscle: a cadaver study
}

\author{
I Ilayperuma ${ }^{1}$, BG Nanayakkara ${ }^{2}$ \\ ${ }^{1}$ Senior Lecturer in Anatomy, ${ }^{2}$ Professor in Anatomy, Department of Anatomy, Faculty of Medicine, \\ University of Ruhuna, Karapitiya, Galle.
}

\begin{abstract}
Introduction: The psoas major (PMA) is a fusiform muscle that covers the anterolateral aspects of the lumbar spine and ends in a tendon which together with the iliacus is inserted into the lesser trochanter of the femur. Racial discrepancies in the anatomy of lumbosacral region, including the psoas muscle have been documented.
\end{abstract}

Objectives: To study the anatomical dimensions of the PMA in Sri Lankan cadavers and to compare the results with those of the white and black cadavers.

Materials and Methods: The anatomical dimensions of the PMA were studied in a total of 34 cadavers with an age span of 54-79 years. The PMA was measured along its entire length. The width of the muscle was measured at each segmental level from L1-S1. The thickness was measured at the level of L4-L5 and anatomical cross sectional area (ACSA) was calculated.

Results: There was a significant gender difference in the ACSA of the PMA. The mean width, thickness and ACSA of the PMA in Sri Lankan cadavers were different from those of the white and black cadavers.

Discussion: The differences in the anatomical dimensions of the PMA in different races may have implications for hip flexor strength, spine function and incidence of low back pathology and warrants further investigation.

\section{Introduction}

The psoas major muscle (PMA) arises from the transverse processes, bodies and intervertebral discs of the twelfth thoracic and five lumbar vertebrae. The muscle proceeds downward across the brim of the lesser pelvis and diminishing gradually in size, passes beneath the inguinal ligament and in front of the capsule of the hip-joint and ends in a tendon which together with the iliacus is inserted into the lesser trochanter of the femur ${ }^{1,2}$.

The PMA contributes to a wide variety of functions. It belongs to a group of muscles called the hip flexors, whose action is primarily to lift the upper leg towards the body when the body is fixed or to pull the body towards the leg when the leg is fixed ${ }^{3}$. The PMA also contributes to lumbar extension ${ }^{4}$, lateral lumbar flexion ${ }^{5}$ and general stabilization of the lumbar spine ${ }^{1,6}$. It also acts as a power source in locomotion ${ }^{7}$.
One of the main causes of lower back dysfunction is the PMA. Because of its attachments along the thoracic and lumbar spine, flexion of the hip causes a pull on the intervertebral discs. This is known as the "psoas paradox" ${ }^{8}$. The PMA has derived this name from its action as a flexor of the hip and spine, while hyperextension of the lumbar spine. Clinically patients with weak abdominal muscles, display spinal hyperextension during a sit-up caused by PMA because of the weak/inadequate antagonist force of the rectus abdominis ${ }^{9}$. A tight, limited psoas will tilt the pelvis anteriorly, creating pressure in the lower back, causing compression and injuries of the intervertebral discs and throwing off gait by limitations in hyperextension at the hip on the push-off phase. The prolapsed intervertebral disc occurs most commonly at the L4 / 5 or L5 / S1 $\operatorname{disc}^{10}$.

It is obvious that the PMA is an important muscle that plays a role in the movements both of the trunk and the lower extremity. 
Furthermore racial differences in the anatomy of lumbosacral region have been established ${ }^{11,12}$. However, only a few studies have investigated the anatomical dimensions and possible anatomical variations of this muscle ${ }^{13}$. Therefore, this study was designed to investigate the possible differences in the anatomical dimensions of the PMA in adult Sri Lankan cadavers.

\section{Materials and methods}

A total of 34 cadavers (22 males and 12 females) that was used for the gross anatomy dissections at the Department of Anatomy, Faculty of Medicine, Galle, Sri Lanka, were used for this study. Subjects were aged between 54 to 79 yrs. There were no visible gross abnormalities in the lumbosacral region of these cadavers.

After incising through the anterior abdominal wall and removing the viscera the psoas major muscle was exposed. The attachments of the muscle were examined and the following measurements were taken. The length of the PMA was measured from the most cranial attachment on the T12 vertebra to the lesser trochanter of the femur. The width of the muscle (diameter of the muscle in the coronal plane) was measured at each segmental level from L1 L2 to L5 - S1. The thickness of the muscle (diameter of the muscle in the sagittal plane) was measured at the segmental level of L4 - L5. Vertebral attachments of the muscle were severed and the muscle was divided in cross section and re-measured to verify the measurements.
The above dimensions of the muscle were measured using a measuring tape and a sliding caliper (Mitutoyo, Japan) capable of measuring to the nearest $0.01 \mathrm{~mm}$. The anatomical cross sectional area (ACSA) of the PMA was calculated according to the method described by Hanson et al. $1999^{13}$. Paired t-test was used to analyze the data. An alpha level of $\mathrm{P}<0.05$ was considered significant.

\section{Results}

The PMA covers the anterolateral aspects of the lumbar spine and inserts on the lesser trochanter of the femur. These attachment sites of the muscle correspond well with those described in the anatomy text books.

The mean length, width, thickness and ACSA of the muscle according to the gender are shown in Table 1. There was a significant variation in the thickness and ACSA of the PMA between males and females. The mean length and width of the PMA did not vary significantly between the sexes.

A comparison of the anatomical dimensions of the PMA in male black and white cadavers ${ }^{13}$ with those of this study is shown in Table 2 . There was a difference between the mean width, thickness and ACSA of the PMA in Sri Lankan cadavers when compared with that of both the white and black cadavers. However, these differences were not statistically compared due to differences in the age distribution of the study group.

Table 1 - Anatomical dimensions (mean \pm SD) of the PMA

\begin{tabular}{lccc}
\hline Dimensions $($ mean \pm SD) & Level & Male & Female \\
\hline Length $(\mathbf{m m})$ & & $288.4 \pm 0.05$ & $263.30 \pm 17.85$ \\
Thickness $(\mathbf{m m})$ & L4-L5 & $24.38 \pm 4.51^{*}$ & $14.12 \pm 4.04$ \\
Width $(\mathbf{m m})$ & L1-L2 & $33.11 \pm 4.51$ & $30.60 \pm 3.21$ \\
& L2-L3 & $44.56 \pm 6.91$ & $40.40 \pm 6.69$ \\
& L3-L4 & $47.67 \pm 7.11$ & $40.80 \pm 6.98$ \\
& L4-L5 & $40.11 \pm 9.14$ & $32.80 \pm 3.56$ \\
& L5-S1 & $32.56 \pm 7.92$ & $25.60 \pm 4.28$ \\
ACSA $\left(\mathbf{m m}^{2}\right)$ & L4-L5 & $778.9 \pm 187.11^{*}$ & $368.02 \pm 126$ \\
\hline \multicolumn{4}{c}{} \\
& &
\end{tabular}


Table 2 - Racial differences (mean \pm SD) in PMA

\begin{tabular}{lcccc}
\hline $\begin{array}{l}\text { Dimensions } \\
(\text { mean } \pm \mathbf{S D})\end{array}$ & Level & $\begin{array}{c}\text { Sri Lankan Male } \\
\text { (our study) }\end{array}$ & $\begin{array}{l}\text { Black Male } \\
\text { (Hanson et al. 99) }\end{array}$ & $\begin{array}{c}\text { White Male } \\
\text { (Hanson et al. 99) }\end{array}$ \\
\hline Thickness (mm) & L4-L5 & $24.38 \pm 4.51$ & $43.9 \pm 1.5$ & $20.8 \pm 1.1$ \\
Width (mm) & L1-L2 & $33.11 \pm 4.51$ & $30.3 \pm 1.6$ & $15.1 \pm 1.2$ \\
& L2-L3 & $44.56 \pm 6.91$ & $40.1 \pm 1.6$ & $22.3 \pm 1.2$ \\
& L3-L4 & $47.67 \pm 7.11$ & $53.8 \pm 2.0$ & $30.2 \pm 1.2$ \\
& L4-L5 & $40.11 \pm 9.14$ & $49.5 \pm 1.8$ & $22.1 \pm 1.2$ \\
& L5-S1 & $32.56 \pm 7.92$ & $37.7 \pm 1.9$ & $17.3 \pm 1.1$ \\
& L4-L5 & $778.9 \pm 257.11$ & $1708.6 \pm 100.1$ & $361.8 \pm 34.0$ \\
\hline
\end{tabular}

\section{Discussion}

To the best of our knowledge morphology and anatomical characteristics of the PMA has not been addressed in an adult Sri Lankan population. Anatomical differences in the PMA have been reported in black and white people ${ }^{13}$. This study describes the anatomical dimensions of the PMA in a total of 34 Sri Lankan cadavers and compares the results with that of black and white people.

Generally speaking the size of a muscle often indicates its strength ${ }^{1}$. The maximum contractile force developed by an active muscle depends on the physiological cross sectional area (PCSA) of the muscle ${ }^{1,14}$. The fiber pennation angle of the PMA is zero degrees ${ }^{15}$. It has been shown that when the pennation angle of a muscle is zero degrees, the PCSA corresponds to the ACSA. Accordingly it is evident that PCSA and ACSA are almost identical for the PMA.

There was a significant difference in the ACSA of PMA between Sri Lankan males (778.9 \pm $\left.187.11 \mathrm{~mm}^{2}\right)$ and females $\left(368.02 \pm 126 \mathrm{~mm}^{2}\right)$. In females the size of PMA was less than half that of the males. These data suggest that gender related changes in size and presumably strength of the PMA is gender specific. Since age of the subjects was in the range of 54 to 79 yrs, this marked reduction in female PMA muscle mass may also be due to post-menopausal hormonal changes. It was interesting to note, that the mean ACSA of our study population was markedly larger and smaller than the dimensions of white and black cadavers, respectively. It has been postulated that black individuals are likely to produce great hip flexion force than white individuals ${ }^{13}$. Therefore, similarly, although unconfirmed, it is likely that Sri Lankans are capable of producing a greater hip flexion force than white individuals. This observation may have functional implications as PMA is the largest hip flexor muscle ${ }^{3}$. It has been shown that iliopsoas is maximally activated during a soccer player's kick. A large and strong PMA may be an advantage in events that require kicking movements, and during sprint running 16. It remains to be established, if such a race specific size difference reflects in the strength of the PMA in these populations.

The function of the PMA as a general stabilizer of the lumbar spine has been well established ${ }^{1}$. Activation of PMA also contributes to ipsilateral lateral bending and contralateral rotation ${ }^{17}$. Furthermore, it can accentuate the lumbar lordosis. PMA has also been suggested as the prime mover of the lumber spine and known to exert large compression and shear loading on the lumbar joints ${ }^{3}$. Therefore, size difference in PMA among races may affect the above functional capacities.

In conclusion, for the first time, the present study addresses the anatomical dimensions of the PMA in a group of adult Sri Lankan cadavers. The results show that the dimensions of PMA are gender and race specific. The differences in the size of the PMA may have implications for hip flexor strength, spine function and incidence of low back pathology. 


\section{Acknowledgement}

The authors wish to thank Dean, Faculty of Engineering, University of Ruhuna, for lending the sliding calipers used in this study, Drs. E. Wannigama, K.L.A. Udenika, R.E. Attanayake, W. Abeygunarathna, H.A.A. Wimal for their help with the project and Mrs. K. N. Palahepitiya for technical assistance.

\section{References}

1. Gray H. et al. Gay's Anatomy. $38^{\text {th }}$ edition, Churchchill Livingston, 2000.

2. Bassmajian J, Grants Method of Anatomy, $11^{\text {th }}$ edition, Baltiomore, Williams and Wilkins, 1989.

3. Bogduk N, Pearcy M, Hadfield G. Anatomy and biomechanics of psoas major. Clinical Biomechanics 1992; 7: 109-15.

4. Rab GT, Chao EY, Stauffer RN. Muscle force analysis of the lumbar spine. Orthopaedic Clinics of North America 1977; 8: 193-9.

5. Rommanes GJ, Cunningham's Textbook of Anatomy, $12^{\text {th }}$ edition, Oxford University Press, 1981.

6. Crisco JJ, Panjabi MM. Postural biomechanical stability and gross muscular architecture in the spine. In Multiple Muscle Systems: Biomechanics and Movement Organization. Springer, New York, 1990.

7. Gracovetsky S, Farfan H. The optimum spine. Spine 1986; 11: 543-73.
8. Rasch PJ, Burke RK. Kinesiology and Applied Anatomy. Philadelphia: Lea \& Febiger 1978.

9. Janda V. Muscle Function Testing. London: Butterworths, 1983.

10. Ellis HA. Clinical Anatomy: Revision and applied anatomy for clinical students, $9^{\text {th }}$ Edition, Blackwell Science.

11. Hanson P, Magnusson SP, Simonsen EB. The sacrum and lumbosacral curve differ in young black and white people. Acta Anatomica 1998; 162: 226-31.

12. Hanson P. The differences in anatomy of the lumber anterior longitudinal ligament in young African Americans and Scandinavians. Archives of Physical Medicine and Rehabilitation 1998; 79:1545-8.

13. Hanson P, Peter MS, Sorensen H, Erik BS. Anatomical differences in the psoas muscles in young black and white men. Journal of Anatomy, 1999; 194: 303-7.

14. Liber RL. Skeletal Muscle and Function. Baltimore. Williams \& Wilkins, 1992.

15. White SC. A deterministic model using EMG and muscle kinematics to predict individual muscle forces during normal human gait. Thesis. Department of Kinesiology, University of Waterloo. 1989.

16. Simonsen EB, Thomsen L, Klausen K. Activity of mono and biarticular leg muscles during sprint running. European Journal of Applied Physiology.1985; 54: 524-32.

17. Kapandji IA. The physiology of the Joints, vol 3, Churchill Livingstone, New York, 1974. 